\title{
SUBCARTESIAN PRODUCTS OF FINITELY MANY FINITE ALGEBRAS 1
}

\author{
AHMAD SHAFAAT
}

ABSTRACT. We show that if the lattice of subquasivarieties of a quasivariety of $\Omega$-algebras has a finite maximal chain then every algebra of the quasivariety is a subcartesian product of a family of certain finitely many finite algebras.

For definitions of common concepts related to $\Omega$-algebras that are used but not defined here we refer the reader to [1]. Throughout this note we shall assume $\Omega$ to be arbitrarily fixed. The letter $\Omega$ will denote an arbitrary class of $\Omega$-algebras.

There are many well-known examples of quasivarieties $\Omega$ of algebras with the following property:

(.) There exists a finite set $\Omega^{\prime}$ of finite algebras in $\Omega$ such that every algebra in $\Omega$ is embeddable in the cartesian product of a family of algebras in $\Re^{\prime}$.

Varieties of semilattices, distributive lattices, abelian groups of exponent $n$, normal idempotent semigroups [7], and the quasivarieties $\mathfrak{A}_{m}$ of unary algebras $\langle A ; f\rangle$ with $f$ satisfying $f^{m}(x)=x, f\left(x_{1}\right)=f\left(x_{2}\right) \rightarrow x_{1}$ $=x_{2}$ are some of these examples. Every one of these quasivarieties has finitely many subquasivarieties and the purpose of this note is to relate this property (of finiteness of the number of subquasivarieties) to $(\cdot)$. More precisely we shall prove

THEOREM 1. For every quasivariety $\Re$ of $\Omega$-algebras the following two properties are equivalent:

(i) Every subquasivariety of $\Omega$ satisfies $(\cdot)$.

(ii) $\Omega$ is a locally finite quasivariety and the lattice $L_{q}(\Omega)$ of subquasivarieties of $\Omega$ satisfies the ascending chain condition.

In [7] it is shown how results like Theorem 1 can be used to obtain some structural results for a certain type of idempotent semigroups. Theorem 1 can also have consequences like the following:

COROLLARY 1. If a locally finite quasivariety $\Re$ has algebras that are

Received by the editors July 16, 1969 and, in revised form, March 3, 1970.

AMS 1968 subject classifications. Primary 0830.

Key words and phrases. Subcartesian product, quasivarieties of universal algebras, locally finite universal algebras, locally finite quasivarieties.

1 This research was supported by the Summer Research Institute of the Canadian Mathematical Congress. 
not residually finite then every maximal chain in the lattice of subquasivarieties of $\Omega$ is infinite.

The above corollary applies to finite-exponent nonabelian nilpotent varieties of groups [5], showing that such varieties have infinitely many subquasivarieties. The simple fact needed here, namely, that for every integer $n>2$ there are nilpotent groups of exponent $n$ that are not residually finite was pointed out to me by Dr. L. G. Kovacs.

The lemmas required for the proof of Theorem 1 have another interesting consequence. Let a class $\Omega$ be called quasiprimitive if $\Omega$ contains subalgebras and cartesian products of its algebras.

THEOREM 2. If a locally finite quasivariely $\Omega$ has finitely many subquasivarieties then every quasiprimitive subclass of $\Omega$ is a subquasivariety.

We now turn to proving the above two results. We need two lemmas.

LEMMA 1. A class $\Omega$ of locally finite $\Omega$-algebras is a quasivariety if and only if $\Omega$ is a local quasiprimitive class.

Proof. Every quasivariety is clearly local and quasiprimitive. Conversely assume that $\Omega$ is a local quasiprimitive class of locally finite algebras. By the proof of Theorem 1 of [6] $\Omega$ can be defined by a set of implications of the form

( $\alpha) \forall x_{1}, \cdots, x_{n}\left\langle\left(w_{1}=w_{1}^{\prime} \wedge \cdots \wedge w_{m}=w_{m}^{\prime} \wedge \cdots\right) \rightarrow w_{0}=w_{0}^{\prime}\right\rangle$

where $w_{m}, w_{m}^{\prime}$ are $\Omega$-words in the finitely many variables $x_{1}, \cdots, x_{n}$. Local finiteness of $\Omega$ can be expressed by a set $\Sigma$ of identities so that using $\Sigma$ we can reduce $(\alpha)$ to a finite implication. Hence $\Omega$ can be defined by $\Sigma$ and finite implications. This proves the lemma.

Our next lemma is known ${ }^{2}[3]$ and is closely related to the main theorem of $[4]$.

Let $Q(\Omega)$ denote the class of algebras embeddable in cartesian products of algebras from $\Omega$. Clearly $\Omega$ is quasiprimitive if and only if $Q(\Re)=\Omega$.

LEMMA 2. If $\Omega$ is a finite class of finite $\Omega$-algebras then $Q(\Omega)$ is local.

Proof of Theorem 1 . Let $\Omega$ satisfy (i) so that $\Omega=Q\left(\Omega^{\prime}\right)$ for some finite class $\Omega^{\prime}$ of finite algebras. By Corollary 3.14 on p. 177 of [1] it follows that the variety $\mathfrak{B}$ generated by $\Omega^{\prime}$ is locally finite. Since

\footnotetext{
${ }^{2}$ I am thankful to Dr. A. F. Pixley for pointing this out to me.
} 
$\Re=Q\left(\Omega^{\prime}\right) \subseteq \mathfrak{B}$ we see that $\Re$ is also locally finite. By Lemma 1 and Lemma 2 it follows that $\Omega$ is a quasivariety. We show that $L_{q}(\Omega)$ satisfies the ascending chain condition. Let $\Omega_{0} \subseteq \Omega_{1} \subseteq \cdots \subseteq \Omega_{l} \subseteq \cdots$ be an ascending chain in $L_{q}(\Omega)$. Write $\Omega_{\infty}=U_{l=0}^{\infty} \Omega_{l}$, where the join is taken in $L_{q}(\Omega)$. We show that $\Omega_{\infty}$ equals the class $\Omega^{+}$of all algebras of $\Omega$ with finite subalgebras in the union $U_{l=0}^{\infty} \Omega_{l}$. Clearly $\Omega^{+} \subseteq \Omega_{\infty}$ because quasivarieties are local and $\Omega$ is locally finite. It is, therefore, enough to show that $\Omega^{+}$is a quasivariety. For this we use Lemma 1 and show that $\Omega^{+}$is a local quasiprimitive class. That $\Omega^{+}$is local and closed under the formation of subalgebras is trivial. The closure under cartesian products follows from: (1) finite cartesian products of finite algebras of $U_{l=1}^{\infty} \Omega_{l}$ are in $U_{l=1}^{\infty} \Omega_{l}$ and (2) a finite subalgebra of the cartesian product of a family of algebras is embeddable in the cartesian product of a finite subfamily. Hence $\Omega^{+}=\Omega_{\infty}$. Now by (i) we can find finite sets $\Omega^{\prime}$ of finite algebras such that $\Omega_{l}=Q\left(\Omega_{l}^{\prime}\right), l=0$, $\cdots, \infty$. By what we have proved about $\Omega_{\infty}$ we can find an integer $l$ such that $\Omega_{\infty}^{\prime} \subseteq \Omega_{l}$, which implies $\Omega_{\infty}=\Omega_{l}$. This proves that (i) implies (ii).

Conversely, let $\Re$ satisfy (ii). By Zorn's lemma and (ii) we can find a finite maximal chain $\Omega_{0} \subset \cdots \subset \Omega_{n}=\Omega$ in $L_{q}(\Omega)$. Since every quasivariety is determined by its finitely generated algebras and since $\Omega_{i}$ is locally finite for $1 \leqq i \leqq n$ we should be able to find a finite algebra $\mathfrak{A}_{i}$ in $\Re_{i}$ which is not in $\Omega_{i-1}$. Write $\Re_{i}^{\prime}=\left\{\mathfrak{A}_{1}, \cdots, \mathfrak{A}_{i}\right\}, 1 \leqq i \leqq n$. By Lemma 1 and Lemma 2, $Q\left(\Omega_{i}^{\prime}\right)$ is a subquasivariety of $\Omega$. We show that $\Omega_{i}=Q\left(\Omega_{i}^{\prime}\right)$. Clearly $\Omega_{0} \subset Q\left(\Omega_{1}^{\prime}\right) \subseteq \Omega_{1}$. By the maximality of the chain $\Omega_{0} \subset \Omega_{1} \cdots C \Omega_{n}$ we see directly that $\Omega_{1}=Q\left(\Omega_{1}^{\prime}\right)$. Continuing this way we arrive at $\Omega_{i}=Q\left(\Omega_{i}^{\prime}\right)$. Hence $\Omega=\Omega_{n}=Q\left(\Omega_{n}^{\prime}\right)$ and (ii) implies $(\cdot)$. Since (ii) is inherited by subquasivarieties (ii) in fact implies (i). This completes the proof.

The above proof has the following consequence.

COROLlARY 2. If $\Omega$ is a locally finite quasivariety such that $L_{q}(\Omega)$ has a finite maximal chain then $\Omega$ satisfies $(\cdot)$.

REMARK. Our proof of Theorem 1 shows one bit more than stated in the theorem; it shows that a class satisfying $(\cdot)$ is a locally finite quasivariety.

Proof OF THEOREM 2 . Let $\Omega^{*}$ be a quasiprimitive subclass of $\Omega$. Let $\Omega_{1}, \cdots, \Omega_{n}$ be all the subquasivarieties of $\Omega$. Clearly, within $\Omega$ the quasivarieties $\Omega_{i}$ are defined by finite sets of implications, say, $\Sigma_{i}$; for otherwise $L_{q}(\Re)$ could not be finite. Let $m$ be the largest number of variables in an implication in $\Sigma_{1} \cup \ldots \cup \Sigma_{n}$. There are only finitely many algebras of $\Omega$ with at most $m$ generators because all 
such algebras are homomorphic images of the free algebra in $\Omega$ with $m$ generators which is finite by the local finiteness of $\Omega$. Let $\mathfrak{A}_{1}, \cdots, \mathfrak{A}_{k}$ be all the algebras (up to isomorphism) in $\mathfrak{\Omega}^{*}$ with at most $m$ generators. We show that $\Omega^{*}=Q\left(\Omega^{\prime}\right)$, where $\Omega^{\prime}$ $=\left\{\mathfrak{A}_{1}, \cdots, \mathfrak{A}_{k}\right\}$. By Lemma 1 and Lemma $2, Q\left(\mathfrak{R}^{\prime}\right)$ is a quasivariety. Let $\Omega_{i}=Q\left(\Omega^{\prime}\right)$. By the choice of $\mathfrak{A}_{1}, \cdots, \mathfrak{A}_{k}$ every implication in at most $m$ variables which holds in $\mathfrak{A}_{1}, \ldots, \mathfrak{A}_{k}$ also holds in all other algebras of $\Omega^{*}$. Since every implication of $\Sigma_{i}$ has at most $m$ variables we see that $\Sigma_{i}$ holds in all algebras of $\Omega^{*}$, so that $\Omega^{*} \subseteq \Omega_{i}$. The opposite inclusion is trivial and hence $\Omega^{*}=\Omega_{i}$. This completes the proof.

\section{REFERENCES}

1. P. M. Cohn, Universal algebra, Harper and Row, New York, 1965. MR 31 \#224. pear).

2. J. A. Gerhard, The lattice of equational classes of idempotent semigroups (to ap-

3. G. Grätzer, On the class of subdirect powers of a finite algebra, Acta. Sci. Math. (Szeged) 25 (1964), 160-168. MR 29 \#5772.

4. B. H. Neumann, Group properties of countable character (to appear).

5. Hanna Neumann, Varieties of groups, Ergebnisse der Mathematik und ihrer Grenzgebiete, Band 37, Springer-Verlag, Berlin and New York, 1967. MR 35 \#6734.

6. Ahmad Shafaat, On implicationally defined classes of algebras, J. London Math. Soc. 44 (1969), 137-140. MR 38 \#5691.

7. - On the structure of certain idempotent semigroups, Trans. Amer. Math. Soc. 149 (1970), 371-378.

Carleton University, Ottawa, Ontario, Canada 